\title{
GABA is an effective immunomodulatory molecule
}

\author{
Zhe Jin $\cdot$ Suresh Kumar Mendu $\cdot$ Bryndis Birnir
}

Received: 2 August 2011/Accepted: 30 November 2011/Published online: 13 December 2011

(C) The Author(s) 2011. This article is published with open access at Springerlink.com

\begin{abstract}
In recent years, it has become clear that there is an extensive cross-talk between the nervous and the immune system. Somewhat surprisingly, the immune cells themselves do express components of the neuronal neurotransmitters systems. What role the neurotransmitters, their ion channels, receptors and transporters have in immune function and regulation is an emerging field of study. Several recent studies have shown that the immune system is capable of synthesizing and releasing the classical neurotransmitter GABA ( $\gamma$-aminobutyric acid). GABA has a number of effects on the immune cells such as activation or suppression of cytokine secretion, modification of cell proliferation and GABA can even affect migration of the cells. The immune cells encounter GABA when released by the immune cells themselves or when the immune cells enter the brain. In addition, GABA can also be found in tissues like the lymph nodes, the islets of Langerhans and GABA is in high enough concentration in blood to activate, e.g., GABA-A channels. GABA appears to have a role in autoimmune diseases like multiple sclerosis, type 1 diabetes, and rheumatoid arthritis and may modulate the immune response to infections. In the near future, it will be important to work out what specific effects GABA has on the function of the different types of immune cells and determine the underlying mechanisms. In this review, we discuss some of the recent findings revealing the role of GABA as an immunomodulator.
\end{abstract}

Z. Jin · S. K. Mendu · B. Birnir $(\bowtie)$

Department of Neuroscience, Molecular Physiology

and Neuroscience, Uppsala University, BMC, BOX 593,

75124 Uppsala, Sweden

e-mail: bryndis.birnir@neuro.uu.se

URL: http://www.neuro.uu.se/forskning/

forskargrupp.html\&id=56
Keywords GABA - GABA-A · Neurotransmitter · Immunomodulation · Autoimmune disease $\cdot$ Immune cells

\section{Introduction}

The role of GABA in a physiological process is best studied in the brain where GABA is the main inhibitory neurotransmitter. GABA is made and released by neurons and it activates GABA-A ion channels and the GABA-B receptor in the neuronal plasma membrane. Activation of the channels and receptor generally results in decreased neuronal excitability in mature neurons. The low extracellular GABA concentration is maintained by reuptake of GABA into the neurons and astrocytes by sodium-dependent GABA cotransporters.

GABA is produced by decarboxylation of the amino acid glutamate by the enzyme glutamic acid decarboxylase (GAD) that exists in two isoforms GAD65 and GAD67. The two GAD isoforms have different subcellular location with GAD67 distributed evenly throughout the neuronal cytoplasm whereas the GAD65 is associated with synaptic vesicles (Buddhala et al. 2009). GABA is metabolized into succinic semialdehyde by the action of the enzyme GABA transaminase (GABA-T).

The GABA-A ion channels are pentameric chloride channels and normally contain three types of subunits: $2 \alpha$, $2 \beta \mathrm{s}$ and a third type of subunit. To date, 19 different mammalian GABA-A subunits have been cloned $(\alpha 1-6$, $\beta 1-3, \gamma 1-3, \delta, \varepsilon, \pi, \theta, \rho 1-3)$ (Olsen and Sieghart 2009). Evidence for the existence of a multitude of GABA-A channel subtypes comes from pharmacological studies. It has been shown that, e.g., benzodiazepine-site ligands can differentiate between GABA-A channel subtypes based on the type of $\alpha$ and $\gamma$ subunits in the channel complex (Olsen 
and Sieghart 2009). The list of GABA-A channel subtypes has been further extended and confirmed with other ligands such as GABA and the general anesthetics (Olsen and Sieghart 2009). All neurons have GABA-A ion channels but the subtypes that are expressed in a neuron change during development and vary between brain regions and the different types of neurons (Laurie et al. 1992; Wisden et al. 1992). The channels are either located at synapses where they generate phasic currents or outside of synapses where they are termed extrasynaptic channels and generate tonic currents (Birnir and Korpi 2007). In contrast, there is only one type of the GABA-B receptor which is a G-protein-coupled receptor composed of two subunits, GABA-B1 and GABA-B2 (Marshall et al. 1999).

In the past, opening of neuronal GABA-A channels was thought to only result in hyperpolarization of the neuronal membrane potential due to low intracellular chloride concentration in neurons. Today we know that, during neuronal development the intracellular chloride concentration decreases resulting in a change from the predominant depolarizing phenotype of immature neurons to the hyperpolarizing phenotype of adult neurons. However, within mature neurons intracellular chloride gradients can exist (Zilberter et al. 2010; Cherubini and Ben-Ari 2011). Whether the activation of GABA-A channels by GABA leads to depolarization or hyperpolarization of the resting membrane potential in cells is therefore related to the intracellular chloride concentration that is regulated by chloride transporters in neurons (Blaesse et al. 2009).

GABA is not only present within the central nervous system (CNS) but has also been identified in many organs such as the pancreas, pituitary, testes, gastrointestinal tract, ovaries, placenta, uterus and adrenal medulla (Gladkevich et al. 2006) and GAD is expressed, at least, in cells in the pancreatic islets, adrenal gland and testes. In recent years, it has become evident that cells of the immune system may also produce GABA and express GABA-A ion channels, GABA transporters and the GABA-B receptor. A misconception that has contributed to the ignorance of the role of GABA outside of the CNS is that it was widely accepted that GABA-A channels were only fully activated by millimolar concentrations of GABA. It is now clear that this is not correct (Birnir et al. 1994; Bai et al. 2001; Semyanov et al. 2003; de Groote and Linthorst 2007) as extrasynaptic channels can be activated by million times lower GABA concentrations than their synaptic counterparts and may be saturated by nano to micromolar GABA concentrations (Lindquist and Birnir 2006; Jin et al. 2011). Importantly, submicromolar GABA concentrations are present around neurons in the brain, within the pancreatic islets and in blood (Petty et al. 1999; Wendt et al. 2004; de Groote and Linthorst 2007; Oresic et al. 2008).

\section{The components of the GABA signaling machinery expressed in immune cells}

The neuronal GABAergic system is composed of four primary parts: the GABA-A channels, the GABA-B receptor, the GABA transporters and the enzymes that make or degrade GABA (Fig. 1). So far, there are relatively few studies that have looked at the GABA system in immune cells (Table 1).

The enzymes responsible for GABA synthesis have been detected in T cells, macrophages and dendritic cells (Bhat et al. 2010; Dionisio et al. 2011). GAD65 was present in dendritic cells and macrophages (Bhat et al. 2010) from encephalomyelitis (EAE) mice whereas GAD67 was detected in human peripheral monocytes (Dionisio et al. 2011). GABA secretion from stimulated mouse macrophages and $\mathrm{T}$ cells has been reported (Bhat et al. 2010; Soltani et al. 2011) and GABA was detected in extracts from human peripheral blood macrophages (Stuckey et al. 2005). GABA-T has been detected in macrophages, $\mathrm{CD}^{+} \mathrm{T}$ cells and peripheral human monocytes (Bhat et al. 2010; Dionisio et al. 2011). In immune cells only one study, so far, has examined the expression of the transporter that transport GABA into synaptic vesicles [vesicular inhibitory amino acid transporter (VIAAT)]. In peripheral human monocytes, gene transcripts were detected for VIAAT and then additionally verified by immunohistochemistry (Dionisio et al. 2011).

Gene transcripts encoding the GABA-A channel subunits have been detected in several types of immune cells but which subunits were present varied. Tian et al. (2004) identified the $\alpha 1, \alpha 2, \beta 1, \beta 2, \gamma 3$ and $\delta$ subunits in $\mathrm{CD}^{+}{ }^{+} \mathrm{T}$ cells from NOD (non-obese diabetic) mice, a mouse model for type 1 diabetes, whereas Bhat et al. (2010) did not identify any of the four subunits $(\alpha 1, \beta 1, \varepsilon, \gamma 2)$ they examined in $\mathrm{CD}^{+}{ }^{+} \mathrm{T}$ cells from EAE mice, the animal model for multiple sclerosis (MS). Only in two studies was the expression of all 19 different GABA-A channel subunits examined; in an EAE cell line, the $\alpha 1, \alpha 4, \beta 2, \beta 3, \gamma 1$ and $\delta$ subunits were detected (Bjurstom et al. 2008) and in $\mathrm{CD} 4^{+}$and $\mathrm{CD} 8^{+} \mathrm{T}$ cells from Wistar rats were the $\alpha 1, \alpha 2, \alpha 3, \alpha 4, \alpha 6, \beta 3, \gamma 1, \delta, \rho 1$ and $\rho 2$ subunits identified out of the 19 subunits (Mendu et al. 2011). It is therefore possible that more subunits types can be detected in $\mathrm{T}$ cells from the mouse models or alternatively, the expression of the GABA-A subunits may be regulated depending on, e.g., the state of activation of the cells or the species. Clearly the $\mathrm{T}$ cells express the GABA-A channel subunits but what determines the specific channel subtypes in the cells remains to be determined. Similarly, the $\alpha 1, \alpha 2$, $\beta 3$ and $\delta$ subunits have been detected in cultured peritoneal macrophages and the $\beta 1$ and $\varepsilon$ subunits in macrophages isolated from the EAE mouse model (Bhat et al. 2010). 
Fig. 1 Schematic figure showing the various components of the GABA signalling system. GABA gamma-aminobutyric acid, VIAAT vesicular inhibitory amino acid transporter, $G A T$ GABA transporter, $G A B A-T$ GABA transaminase, $G A D$ glutamic acid decarboxylase, SSA succinic semialdehyde

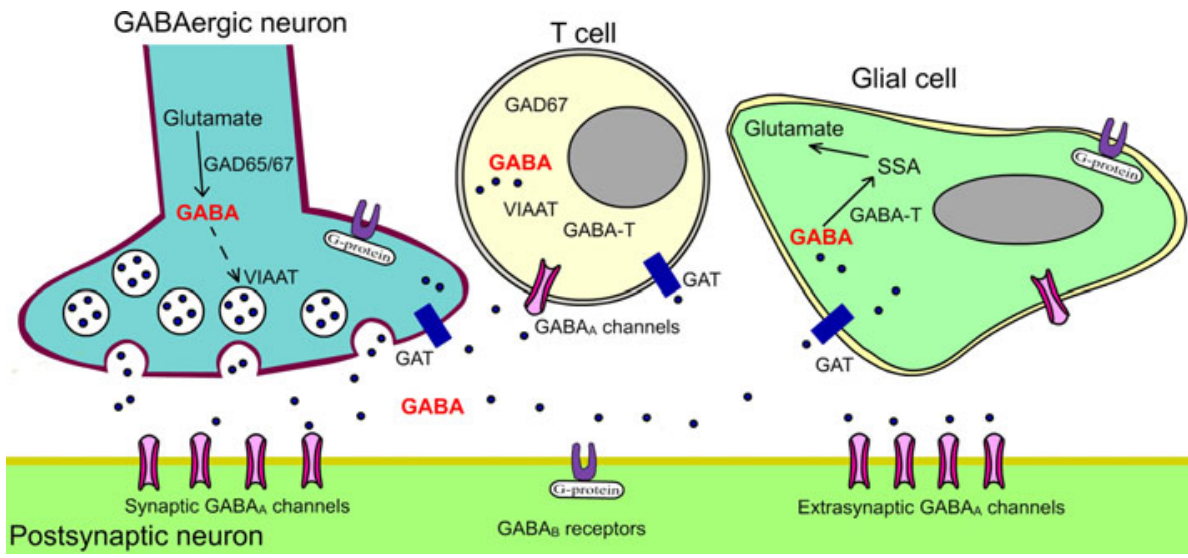

Human peripheral monocytes expressed the $\alpha 1, \alpha 3, \beta 2$ and the $\delta$ subunits (Alam et al. 2006). So far, other immune cells like dendritic cells or natural killer cells have not been reported to express GABA-A channels subunits. Together these results demonstrate that immune cells from rats, mice and humans do have the necessary building blocks to form GABA-A ion channels. Whether the GABA-B receptor is of importance for the immunological effect of GABA on immune cells is not known today.

There have been four plasma membrane GABA cotransporters identified (GAT1-4) in neurons. All of these cotransporters mediate ion-coupled secondary active transport of GABA across the plasma membrane. Only two of these cotransporters have, so far, been detected in immune cells. Gene transcripts for the GAT-1 or GAT-2 have been identified in human peripheral lymphocytes (Dionisio et al. 2011) and in T cells from EAE mice but the results differ in whether a transcript is detected in resting (Bhat et al. 2010) or in activated cells (Wang et al. 2008). Interestingly, both Wang et al. (2008) and Bath et al. (2010) isolated macrophages form the EAE mouse model but only Bath et al. (2010) detected GABA cotransporter in these cells and then only in stimulated macrophages.

The components of the GABA signaling system appear to be dynamically regulated in immune cells as is reflected by the variability in results from the few studies published to date. Whether the results also depend on the species (humans, rats and mice), strains of animals or animal models, state of activation of the cells or classes of cells or different subtypes of GABA-A ion channels and GABA transporters expressed in the cells remains to be examined.

\section{Effects of GABA on immune cells}

Bergeret et al. (1998) reported that GABA modulated cytotoxicity of immunocompetent cells expressing GABA$A$ ion channel subunits. GABA activated GABA-A ion channel currents in $\mathrm{T}$ cells and macrophages (Bjurstom et al. 2008; Bhat et al. 2010; Mendu et al. 2011) and GABA application resulted in decreased cytokine secretion and $\mathrm{T}$ cells proliferation (Tian et al. 2004; Bjurstom et al. 2008; Mendu et al. 2011) or had no effects on these properties of the cells (Bhat and Steinman 2009). In lymphocytes, exposure to GABA reduced but did not abolish the transient increase in the intracellular calcium concentration that was associated with activation of the cells (Alam et al. 2006). Drugs that mimic GABA (e.g. muscimol) or increase the ambient GABA concentration in the brain (e.g. vigabatin, gabaculine) decreased cytokine production in macrophages (Reyes-Garcia et al. 2007; Bhat et al. 2010). The GABA transporters have also been reported to modulate cytokine production and $\mathrm{T}$ cell proliferation. In GAT-1 knock-out mice both cell proliferation and IFN- $\gamma$ production are increased in $\mathrm{T}$ cells from these animals relative to T cells from wild-type mice (Wang et al. 2008). Finally, in classical immunological chemotaxis and phagocytotic assays, pharmacological modulation of human peripheral monocytes with drugs acting at the GABA-A channels impaired the function of the cells (Wheeler et al. 2011). The GABA signaling system is clearly active in the immune cells and can affect a variety of functional properties of the cells like cytokine secretion, cell proliferation, phagocytic activity and chemotaxis. Nevertheless, much remains to be discovered, as we know relatively little about how these processes are linked to GABA and the GABA signalling system in the cells.

\section{Role of GABA in immune and autoimmune diseases}

GABA is decreased in the serum of MS patients (Demakova et al. 2003). Oral GABA treatment down-regulated inflammatory responses in a mouse model of rheumatoid arthritis and in a mouse model of obesity (Tian et al. 2011a, 







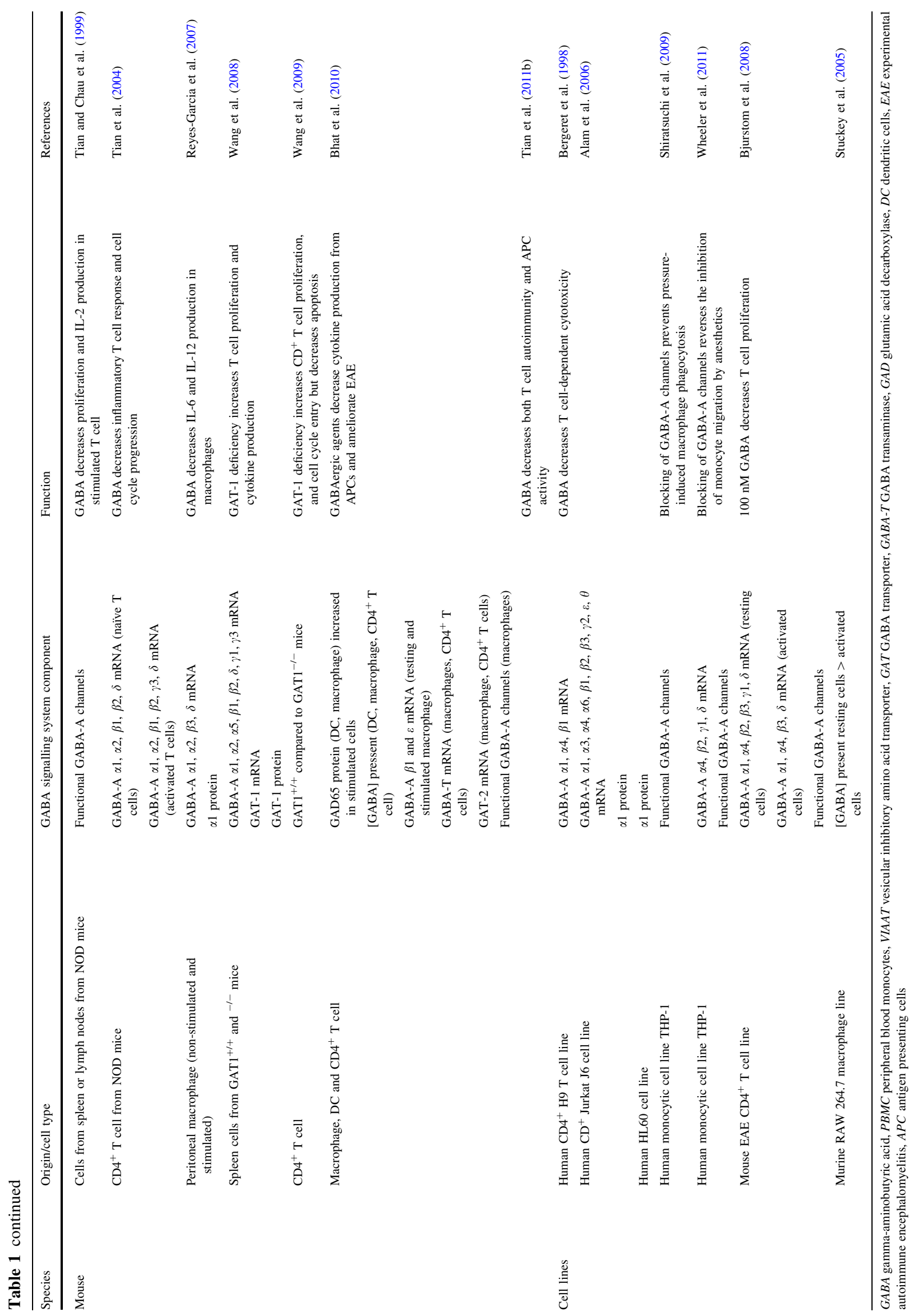


2011b). Disregulation of the GABA metabolism preceded pancreatic islets autoimmunity in children who later progressed to type 1 diabetes (Oresic et al. 2008) and GAD is a major autoantigen in type 1 diabetes (Lernmark et al. 1978). What role the GABA system has in these diseases remains to be clarified. The realization that extrasynaptic GABA-A ion channels in immune cells can be fully activated by submicromolar GABA concentrations makes GABA a potential effector molecule in many parts of the body including blood, pancreatic islets, cerebrospinal fluid and, of course, in the brain where the ambient GABA concentration is in the submicromolar range (Tossman et al. 1986; de Groote and Linthorst 2007). Tian et al. (2004) showed that increasing the systemic GABA concentration delayed the onset and incidence of type 1 diabetes in NOD/scid mice. Similarly, Bath et al. (2010) showed that increasing the ambient CNS GABA concentration ameliorated ongoing paralysis in EAE mice, by inhibiting onset of inflammation. GABA has also been proposed to have a role in rheumatoid arthritis (Kelley et al. 2008; Tian et al. 2011b) and psoriasis (Nigam et al. 2010). The cross-talk between the immune cells and the affected tissues is complex but the relatively few studies published to date imply that GABA and the GABA signaling system components are an important part of the disease environment.

\section{Conclusion}

In recent years, it has become evident that cells of the immune system are capable of synthesizing and releasing the classical neurotransmitter GABA and they do often express proteins that are parts of the neuronal GABA signalling system. Immune cells from rats, mice and humans do have the necessary building blocks to form GABA-A ion channels. The absence of a presynaptic terminal defines these channels in the immune cells as extrasynaptic-like channels. Physiologically this seems reasonable as the cells will not encounter the same high millimolar concentrations of GABA as in the neuronal synapses but rather will be exposed to submicromolar GABA concentrations as they travel in the blood, enter the brain or the pancreatic islets. When GABA opens the GABA-A channels in the plasma membrane of immune cells, the membrane potential changes and thereby a number of cellular processes may be affected such as, e.g., calcium entry into the cell. Despite the limited number of studies to date, there are significant differences between the results such as what GABA-A channel subunits and therefore what channel subtypes are expressed in the immune cells. How easily the channels change the membrane potential is related to the GABA-A channel subtype. The subtypes differ in terms of efficiency of ion conduction as the single-channel conductance and kinetic properties vary (Lindquist and Birnir 2006; Olsen and Sieghart 2009). Furthermore, properties like the affinity for GABA and modulation by drugs such as the benzodiazepines and steroids is determined by the specific subunit composition of the GABA-A ion channel subtype (Lindquist and Birnir 2006; Olsen and Sieghart 2009). It is, therefore, of great importance to know what subunits do form the channel and how their composition is determined and regulated in the immune cells. The discrepancies in results between studies may reflect difference between species (humans, rats and mice) or some experimental condition such as different strains of animals or animal models, different state of activation of the immune cells or classes of cells and in some cases, limited number of subunits being examined.

GABA appears to have a role in autoimmune diseases like MS, type 1 diabetes and rheumatoid arthritis and may modulate the immune response to infections (Bhat et al. 2010; Mendu et al. 2011; Soltani et al. 2011; Tian et al. 2011b; Wheeler et al. 2011). Neuroinflamation is involved in epileptogenic process in the brain (Vezzani et al. 2008) and has even a role in Alzheimer disease, stroke and traumatic brain injury (Popovich and Longbrake 2008; Schwartz and Shechter 2010). Clearly there is an extensive cross-talk between the immune and the nervous system and one of the molecules participating in the exchange between the two systems is GABA. Despite the few studies, it is apparent that the GABA signaling system is an important and integral part of the immune system. In the near future, it will be important to work out what determines whether components of the GABA system are expressed or not in an immune cell and then, what cellular processes are affected when GABA activates GABA-A channels, GABA transporters and the GABA-B receptor in the plasma membrane of the cells. Identifying the GABA-A channel subtypes expressed in the immune cells may be of great clinical significance as a number of drugs modulating functional properties of GABA-A channels are widely used in clinical settings.

Acknowledgments We thank the Swedish Research Council, Uppsala University, EXODIAB (consortium for Excellence of Diabetes Research in Sweden), the Ernfors Foundation and the Swedish Diabetes Foundation for financial support. Zhe Jin holds a postdoctoral fellowship from SSMF (Svenska Sälskapet for Medicinsk Forskning).

Conflict of interest The authors declare that they have no conflict of interest.

Open Access This article is distributed under the terms of the Creative Commons Attribution Noncommercial License which permits any noncommercial use, distribution, and reproduction in any medium, provided the original author(s) and source are credited. 


\section{References}

Alam S, Laughton DL et al (2006) Human peripheral blood mononuclear cells express GABAA receptor subunits. Mol Immunol 43(9):1432-1442

Bai D, Zhu G et al (2001) Distinct functional and pharmacological properties of tonic and quantal inhibitory postsynaptic currents mediated by gamma-aminobutyric acid(A) receptors in hippocampal neurons. Mol Pharmacol 59(4):814-824

Bergeret M, Khrestchatisky $M$ et al (1998) GABA modulates cytotoxicity of immunocompetent cells expressing GABAA receptor subunits. Biomed Pharmacother 52(5):214-219

Bhat R, Axtell R et al (2010) Inhibitory role for GABA in autoimmune inflammation. Proc Natl Acad Sci USA 107(6): 2580-2585

Bhat R, Steinman L (2009) Innate and adaptive autoimmunity directed to the central nervous system. Neuron 64(1):123-132

Birnir B, Everitt AB et al (1994) Characteristics of GABAA channels in rat dentate gyrus. J Membr Biol 142(1):93-102

Birnir B, Korpi ER (2007) The impact of sub-cellular location and intracellular neuronal proteins on properties of GABA(A) receptors. Curr Pharm Des 13(31):3169-3177

Bjurstom H, Wang J et al (2008) GABA, a natural immunomodulator of T lymphocytes. J Neuroimmunol 205(1-2):44-50

Blaesse P, Airaksinen MS et al (2009) Cation-chloride cotransporters and neuronal function. Neuron 61(6):820-838

Buddhala C, Hsu CC et al (2009) A novel mechanism for GABA synthesis and packaging into synaptic vesicles. Neurochem Int 55(1-3):9-12

Cherubini E, Ben-Ari Y (2011) The immature brain needs GABA to be excited and hyper-excited. J Physiol 589(Pt 10):2655-2656

de Groote L, Linthorst AC (2007) Exposure to novelty and forced swimming evoke stressor-dependent changes in extracellular GABA in the rat hippocampus. Neuroscience 148(3):794-805

Demakova EV, Korobov VP et al (2003) Determination of gammaaminobutyric acid concentration and activity of glutamate decarboxylase in blood serum of patients with multiple sclerosis. Klin Lab Diagn 4:15-17

Dionisio L, Jose De Rosa M et al (2011) An intrinsic GABAergic system in human lymphocytes. Neuropharmacology 60(2-3): 513-519

Duthey B, Hubner A et al (2010) Anti-inflammatory effects of the GABA(B) receptor agonist baclofen in allergic contact dermatitis. Exp Dermatol 19(7):661-666

Gladkevich A, Korf J et al (2006) The peripheral GABAergic system as a target in endocrine disorders. Auton Neurosci 124(1-2):1-8

Jin $\mathrm{Z}$, Jin $\mathrm{Y}$ et al (2011) Insulin reduces neuronal excitability by turning on $\mathrm{GABA}(\mathrm{A})$ channels that generate tonic current. PLoS One 6(1):e16188

Kelley JM, Hughes LB et al (2008) Does gamma-aminobutyric acid (GABA) influence the development of chronic inflammation in rheumatoid arthritis? J Neuroinflamm 5:1

Laurie DJ, Seeburg PH et al (1992) The distribution of 13 GABAA receptor subunit mRNAs in the rat brain. II. Olfactory bulb and cerebellum. J Neurosci 12(3):1063-1076

Lernmark A, Freedman ZR et al (1978) Islet-cell-surface antibodies in juvenile diabetes mellitus. N Engl J Med 299(8):375-380

Lindquist CE, Birnir B (2006) Graded response to GABA by native extrasynaptic GABA receptors. J Neurochem 97(5):1349-1356

Marshall FH, Jones KA et al (1999) GABAB receptors: the first 7TM heterodimers. Trends Pharmacol Sci 20(10):396-399

Mendu SK, Akesson L et al (2011) Increased GABA(A) channel subunits expression in $\mathrm{CD} 8(+)$ but not in $\mathrm{CD} 4(+) \mathrm{T}$ cells in $\mathrm{BB}$ rats developing diabetes compared to their congenic littermates. Mol Immunol 48(4):399-407
Nigam R, El-Nour H et al (2010) GABA and GABA(A) receptor expression on immune cells in psoriasis: a pathophysiological role. Arch Dermatol Res 302(7):507-515

Olsen RW, Sieghart W (2009) GABA A receptors: subtypes provide diversity of function and pharmacology. Neuropharmacology 56(1):141-148

Oresic M, Simell S et al (2008) Dysregulation of lipid and amino acid metabolism precedes islet autoimmunity in children who later progress to type 1 diabetes. J Exp Med 205(13):2975-2984

Petty F, Fulton M et al (1999) Evidence for the segregation of a major gene for human plasma GABA levels. Mol Psychiatry 4(6): 587-589

Popovich PG, Longbrake EE (2008) Can the immune system be harnessed to repair the CNS? Nat Rev Neurosci 9(6):481-493

Rane MJ, Gozal D et al (2005) Gamma-amino butyric acid type B receptors stimulate neutrophil chemotaxis during ischemiareperfusion. J Immunol 174(11):7242-7249

Reyes-Garcia MG, Hernandez-Hernandez $F$ et al (2007) GABA (A) receptor subunits RNA expression in mice peritoneal macrophages modulate their IL-6/IL-12 production. J Neuroimmunol 188(1-2):64-68

Schwartz M, Shechter R (2010) Systemic inflammatory cells fight off neurodegenerative disease. Nat Rev Neurol 6(7):405-410

Semyanov A, Walker MC et al (2003) GABA uptake regulates cortical excitability via cell type-specific tonic inhibition. Nat Neurosci 6(5):484-490

Shiratsuchi H, Kouatli Y et al (2009) Propofol inhibits pressurestimulated macrophage phagocytosis via the GABAA receptor and dysregulation of p130cas phosphorylation. Am J Physiol Cell Physiol 296(6):C1400-C1410

Soltani N, Qiu H et al (2011) GABA exerts protective and regenerative effects on islet beta cells and reverses diabetes. Proc Natl Acad Sci USA 108(28):11692-11697

Stuckey DJ, Anthony DC et al (2005) Detection of the inhibitory neurotransmitter GABA in macrophages by magnetic resonance spectroscopy. J Leukoc Biol 78(2):393-400

Tian J, Chau C et al (1999) GABA(A) receptors mediate inhibition of T cell responses. J Neuroimmunol 96(1):21-28

Tian J, Dang $\mathrm{HN}$ et al (2011) Oral treatment with gammaaminobutyric acid improves glucose tolerance and insulin sensitivity by inhibiting inflammation in high fat diet-fed mice. PLoS One 6(9):e25338

Tian J, Lu Y et al (2004) Gamma-aminobutyric acid inhibits T cell autoimmunity and the development of inflammatory responses in a mouse type 1 diabetes model. J Immunol 173(8):5298-5304

Tian J, Yong J et al (2011) Oral GABA treatment downregulates inflammatory responses in a mouse model of rheumatoid arthritis. Autoimmunity 44:465-470

Tossman U, Jonsson G et al (1986) Regional distribution and extracellular levels of amino acids in rat central nervous system. Acta Physiol Scand 127(4):533-545

Vezzani A, Balosso S et al (2008) The role of cytokines in the pathophysiology of epilepsy. Brain Behav Immun 22(6): 797-803

Wang Y, Feng D et al (2008) Gamma-aminobutyric acid transporter 1 negatively regulates $\mathrm{T}$ cell-mediated immune responses and ameliorates autoimmune inflammation in the CNS. J Immunol 181(12):8226-8236

Wang Y, Luo Q et al (2009) Gamma-aminobutyric acid transporter 1 negatively regulates $\mathrm{T}$ cell activation and survival through protein kinase $\mathrm{C}$-dependent signaling pathways. J Immunol 183(5):3488-3495

Wendt A, Birnir B et al (2004) Glucose inhibition of glucagon secretion from rat alpha-cells is mediated by GABA released from neighboring beta-cells. Diabetes 53(4):1038-1045 
Wheeler DW, Thompson AJ et al (2011) Anaesthetic impairment of immune function is mediated via GABA(A) receptors. PLoS One 6(2):e17152

Wisden W, Laurie DJ et al (1992) The distribution of 13 GABAA receptor subunit mRNAs in the rat brain. I. Telencephalon, diencephalon, mesencephalon. J Neurosci 12(3):1040-1062
Zilberter Y, Zilberter T et al (2010) Neuronal activity in vitro and the in vivo reality: the role of energy homeostasis. Trends Pharmacol Sci 31(9):394-401 